\title{
Elevated Proportion of Small, Dense Low-Density Lipoprotein Particles and Lower Adiponectin Blood Levels Predict Early Structural Valve Degeneration of Bioprostheses
}

\author{
Rahul Shettya,d Nicolas Girerd ${ }^{c}$ Nancy Côté ${ }^{a}$ Benoit Arsenault ${ }^{c}$ \\ Jean-Pierre Després ${ }^{b}$ Philippe Pibarot ${ }^{c}$ Patrick Mathieu ${ }^{a, d}$ \\ a Laboratoire d'Études Moléculaires des Valvulopathies, Groupe de Recherche en Valvulopathies, Quebec Heart

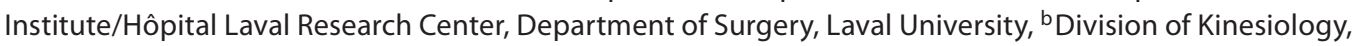 \\ Department of Social and Preventive Medicine, Laval University, and Quebec Heart Institute/Hôpital Laval \\ Research Center, ${ }^{C}$ Hôpital Laval Research Center and Department of Medicine, Laval University, and \\ ${ }^{\mathrm{d}}$ Department of Surgery, Laval University, Québec, Que., Canada
}

\section{Key Words}

Bioprostheses - Structural valve degeneration - Lipid profile $\cdot$ Small, dense low-density lipoprotein · Adiponectin

\begin{abstract}
Objectives: Long-term durability of bioprosthetic heart valves (BPs) are limited by structural valve degeneration (SVD) leading to stenosis and/or regurgitation. In this study, we sought to determine the metabolic markers associated with SVD. Methods: In a cohort of 220 patients with an aortic BP (mean follow-up of $2.5 \pm 1.2$ years), we compared the metabolic and blood lipid profile including the levels of adiponectin and the proportion of small, dense low-density lipoprotein $(\mathrm{LDL})$ particles $\left(\% \mathrm{LDL}_{<255 \AA}\right)$ in individuals developing echocardiographic evidence of early BP hemodynamic dysfunction with subjects having no features of BP dysfunction. Results: Patients developing BP dysfunction $(n=69$; $31.3 \%$ ) had a tendency of higher triglyceride levels. Moreover, patients with BP dysfunction had an increased proportion of $\% L_{L} L_{<255 \AA}$. In multivariate linear regression analysis, after adjustment for age, gender, BP size and hypertension,
\end{abstract}

the $\% L_{L} L_{<255 \AA}(p=0.04)$ was significantly associated with BP dysfunction. In addition, patients with an elevated level of $\% L L_{<255 \AA}$ along with a decreased plasma adiponectin level were at a very high risk of developing early BP hemodynamic dysfunction ( $O R=2.54, p=0.04$ ). Conclusion: BP dysfunction is significantly associated with an increased proportion of small, dense LDL.

Copyright @ 2012 S. Karger AG, Basel

\section{Introduction}

Valve bioprostheses (BPs) are widely used to replace native heart valves with end-stage pathology. Although having a good hemodynamic profile as well as a low thrombogenicity, generally obviating the need for anticoagulation, BPs are still constrained by their limited durability, mostly related to structural valve degeneration (SVD). This process is accelerated in younger patients and could occur due to progressive structural modifications, leading to calcific and non-calcific changes within the valves [1]. Among the various contributors to SVD, 
mechanisms related to pre-implantation fixation as well as immunologic responses against xenoantigens have been documented [2]. Of note, studies have also highlighted that atherosclerotic risk factors, such as hypercholesterolemia, diabetes and metabolic syndrome, were significantly associated with SVD [3-5]. In the same line, recent investigations have documented the presence of lipids and foam cells, a hallmark of atherosclerosis, in explanted BPs for dysfunction [6]. Taken together, these studies support the role of metabolic factors in SVD of BPs. Hence, the objectives of this study were to examine the associations between metabolic markers of atherosclerosis such as the proportion of small, dense low-density lipoprotein (LDL) and adiponectinemia with SVD.

\section{Materials and Methods}

\section{Patient Population}

Between August 1997 and June 2008, a total of 2,117 patients underwent aortic valve replacement with a BP valve at the Quebec Heart and Lung Institute (QHLI). Of this group, 220 patients who had their routine Doppler echocardiographic follow-up performed at the QHLI were included in this study. These patients also had a minimum of two echocardiographic examinations separated by at least 6 months as well as blood plasma available for further analysis of blood markers. The first echocardiographic examination was recorded 1 month after the implantation. Patients with early signs of prosthetic dysfunction 1 month after the surgery were excluded from this study, since it is more likely related to technical issues. Patients who were entered in the study had complete echocardiographic analyses with appropriate acoustic windows. The mean follow-up period was $2.5 \pm 1.2$ years. The study was approved by the QHLI ethical committee.

\section{Doppler Echocardiography}

Echocardiographic analyses were performed by the same technician and reviewed by the attending echocardiographer. Maximum transprosthetic flow velocity was determined by continuouswave Doppler. The mean transprosthetic gradient was calculated using the modified Bernoulli equation. Annualized change in mean gradient ( $\mathrm{mm} \mathrm{Hg}$ /year) was calculated by dividing the difference between the first and last measurements of the follow-up by the time between examinations. Transprosthetic regurgitation was graded semi-quantitatively by color Doppler echocardiography according to the following scale: 0 , none; 0.5 , trivial; 1 , mild; 2 , moderate; and 3 , severe. Worsening of valve regurgitation was defined as an increase of at least 1 degree in the severity of regurgitation during follow-up compared with the value at baseline. We determined the number of patients who had a deterioration of their valve's hemodynamic function during follow-up. This combined variable was defined as a rate of increase in mean gradient of $3 \mathrm{~mm}$ $\mathrm{Hg} /$ year and/or 1 degree worsening of aortic regurgitation [5, 7].

\section{Clinical, Laboratory and Operative Data}

Clinical data including age, gender, history of smoking and documented diagnoses of hypertension, diabetes, hypercholester- olemia, coronary artery disease and renal insufficiency were recorded. Furthermore, fasting plasma lipid profile and glycemia were measured in all patients before surgery. Operative data as well as information on statin treatment were also recorded.

\section{Measurement of LDL Particle Size}

Blood plasma of 220 patients was analyzed. In order to measure LDL particle size, non-denaturing $2-16 \%$ polyacrylamide gradient gel electrophoresis was performed on whole plasma kept at $-80^{\circ} \mathrm{C}[8,9]$. Gels were made with acrylamide and bis-acrylamide (37.5:1) from Bio-Rad (Hercules, Calif., USA) and were cast in our laboratory. A volume of $3 \mu \mathrm{l}$ of whole plasma and $3 \mu \mathrm{l}$ of sample buffer made from sucrose (20\%) and bromophenol blue $(0.25 \%)$ was loaded onto the gels. Electrophoresis was performed in a refrigerated cell $\left(4^{\circ} \mathrm{C}\right)$ for a pre-run of $15 \mathrm{~min}$ at $125 \mathrm{~V}$. This pre-run preceded electrophoresis of the plasma samples at $70 \mathrm{~V}$ for $20 \mathrm{~min}$ and at $150 \mathrm{~V}$ for $4 \mathrm{~h}$. Gels were stained for lipids overnight in a Sudan black solution $(7 \mathrm{~g} / 100 \mathrm{ml})$. Destaining of the gels was performed in a $20 \%$ ethanol solution for $24 \mathrm{~h}$, and original gel size was restored overnight in a $4 \%$ methanol, $0.8 \%$ acetic acid solution. Gels were then scanned using an Alpha Imager scanner from Fisher Scientific (Nepean, Ont., Canada) and were analyzed with the Imagemaster 1D Prime version 4.10 computer software (Amersham Pharmacia Biotech). LDL size was extrapolated from the relative migration of three plasma standards of known diameters (238.3-274.2 $\AA$ ). The estimated diameter for the major peak of each sample was identified as the LDL peak particle size. The relative proportion of LDL with a diameter $<255 \AA$ was ascertained by computing the relative area of the densitometric scan $<255 \AA$ A. It has been documented that Sudan black mainly stains non-polar lipids [10]. The absorbance profile with Sudan black staining was also assumed to closely reflect the cholesterol distribution among LDL particles of different sizes [11].

\section{Measurement of Adiponectin Level}

Human adiponectin serum concentrations from the 220 patients were analyzed and measured by enzyme-linked immunosorbent assay according to the protocol provided by the manufacturer (Human Adiponectin ELISA Kit, B-Bridge International, Inc., Calif., USA).

\section{Statistical Analyses}

Continuous variables were expressed as the mean \pm SD and were compared using $t$ tests for independent samples or one-way ANOVA test as appropriate. When the ANOVA test was used, specific differences between 2 groups were tested with Fisher's least significant difference post hoc test. Differences in proportion were compared using a $\chi^{2}$ test or Fisher's exact test when appropriate. When not normally distributed, the variables were logtransformed to be compared with parametric univariate or multivariate tests. Geometric means were provided when appropriate. Multivariate binary logistic regression was used to identify the independent predictors of prosthesis degeneration. Age, sex and all variables associated with a p value $<0.25$ on univariate analysis were entered into the logistic model. We then constructed another model to separately determine the predictors of prosthesis stenosis and prosthesis regurgitation using multinomial logistic regression. A probability value of $<0.05$ was considered significant. All statistical analyses were performed with SPSS 15 (SPSS Inc., Chicago, Ill., USA). 


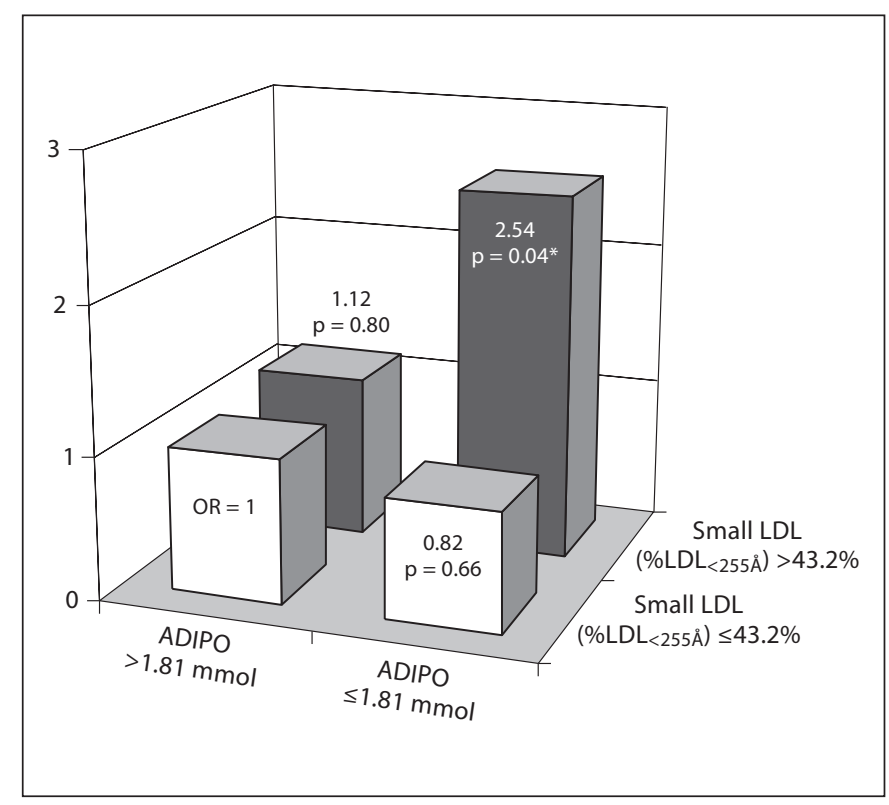

Fig. 1. Interaction between small, dense LDL particles and adiponectin (ADIPO) blood levels in terms of developing progressive structural valve failure in patients with BPs after adjustment for age, gender, body mass index, hypertension and size of BPs (19-21 $\mathrm{mm})$. * $\mathrm{p}$ value compared to low proportion of small, dense LDL and high levels of adiponectin (ADIPO).

Table 1. Manufacturer label

\begin{tabular}{lr}
\hline Type of valve & $\mathrm{n}$ \\
\hline Carpentier Edwards pericardial & 49 \\
Cyrolife O’Brien & 1 \\
Freestyle & 38 \\
Magna & 41 \\
Mitroflow & 18 \\
Mosaic & 72 \\
St. Jude Toronto SPV & 1 \\
\hline Total & 220 \\
\hline
\end{tabular}

\section{Results}

During the follow-up (between the first and last echocardiography), 69 patients (31\%) presented signs of new echocardiographic SVD. The profiles of implanted valves were documented according to manufacturer labels (table 1). Patients with and without SVD had similar clinical characteristics ( $p>0.1$; table 2). However, patients with SVD tended to have higher triglyceride (TG) levels, with
TG $>1.7 \mathrm{mmol} / \mathrm{l}$ (median value) in $33.9 \%$ of patients clinically presenting with features of SVD versus $22.0 \%$ in patients without SVD $(p=0.07)$. There was a trend for an increased proportion of small, dense LDL in patients with SVD [\%LDL $<255 \AA$ $>43.2 \%$ (median value) observed in $59.7 \%$ of patients with SVD vs. $47.6 \%$ in patients without $\operatorname{SVD}(\mathrm{p}=0.10)]$.

After adjusting for age, gender, body mass index, hypertension and prosthesis size, a high proportion of small, dense LDL particles in blood plasma was an independent predictor for higher risk of SVD [OR $=1.89$ for a blood level higher than the median $(>43.2 \%) ; \mathrm{p}=0.04$ ] (table 3).

When adjusting for the same variables, high levels of TG tended to be associated with a higher risk of SVD $(\mathrm{OR}=2.0, \mathrm{p}=0.053)$.

\section{Interactions between Small, Dense LDL Particles and} Adiponectin Blood Levels for the Development of SVD

To test if adiponectin blood levels had a differential impact in patients with low or high levels of small LDL particles, we divided our population into 4 groups. Compared to patients with low levels of small LDL particles and high levels of adiponectin, only patients with high levels of small, dense LDL particles ( $>43.2 \%)$ and low levels of adiponectin $[\leq 1.81 \mathrm{mmol} / \mathrm{l}$ (median value)] had a significantly increased risk of SVD $(\mathrm{OR}=2.54, \mathrm{p}=0.04)$ (fig. 1).

\section{Differential Impact of Metabolic Markers on Clinical}

Presentation as Regurgitation or Stenotic Type of SVD

On univariate analysis, when compared to subjects without SVD, patients with features of SVD, presenting as a new significant isolated regurgitation during follow-up, tended to have lower levels of plasma adiponectin (1.41 \pm 2.60 vs. $1.80 \pm 2.04 \mathrm{mmol} / \mathrm{l} ; \mathrm{p}=0.08$ ). Proportions of small, dense LDL were not different between groups with either isolated regurgitation or significant development of stenosis.

On multivariate analysis, after adjusting for age, gender, body mass index and prosthesis size, lower levels of adiponectin tended to be associated with a higher risk of development of significant and isolated regurgitation during follow-up $(\mathrm{OR}=0.62$ for a 1 -unit increase in adiponectin on a logarithmic scale; $p=0.06$ ), whereas no such association existed for stenotic SVD $(\mathrm{OR}=1.02, \mathrm{p}=0.94)$. Similar associations were observed when further adjusting for plasma $\% \mathrm{LDL}_{<255 \AA}(\mathrm{OR}=0.61, \mathrm{p}=0.06)$ for regurgitant SVD versus $(\mathrm{OR}=1.02, \mathrm{p}=0.93)$ stenotic SVD (table 4). 
Table 2. Profile of patients with BP heart valves

\begin{tabular}{lccc}
\hline & No SVD $(\mathrm{n}=151)$ & SVD $(\mathrm{n}=69)$ & p value \\
\hline Age, years & $71.21 \pm 7.75$ & $71.09 \pm 7.87$ & 0.91 \\
Age $>70$ years, \% & 55.6 & 55.1 & 0.93 \\
Females, $\mathrm{n}$ & $54(35.8)$ & $27(39.1)$ & 0.63 \\
Males, $\mathrm{n}$ & $97(64.2)$ & $42(60.9)$ & \\
Body mass index $>30, \%$ & 27.8 & 18.8 & 0.15 \\
Hypertension, mm Hg & $87(57.6)$ & $46(66.7)$ & 0.20 \\
History of diabetes, $\mathrm{n}$ & $37(24.5)$ & $21(30.4)$ & 0.35 \\
Size of BPs $19-21$ mm, \% & 17.9 & 24.6 & 0.25 \\
High-density lipoprotein level, mmol/l & $1.29 \pm 0.38$ & $1.26 \pm 0.35$ & 0.58 \\
LDL level, mmol/l & $2.43 \pm 0.74$ & $2.46 \pm 0.89$ & 0.81 \\
TG level, mmol/l & $1.40 \pm 0.68$ & $1.51 \pm 0.74$ & 0.30 \\
Median TG $>1.7$ mmol/l, \% & 22.0 & 33.9 & 0.07 \\
Small, dense LDL $(\% L D L<255 \AA)$ & $43.12 \pm 21.35$ & $46.37 \pm 21.63$ & 0.30 \\
\%LDL $<25 \AA$ higher than median value of $43 \%, \%$ & 47.6 & 59.7 & 0.10 \\
Glucose blood level, mmol/l & $6.12 \pm 1.88$ & $5.86 \pm 1.55$ & 0.31 \\
Creatinine clearance, ml/min & $50.64 \pm 18.35$ & $48.04 \pm 15.33$ & 0.31 \\
Adiponectin, mmol/l & $1.79 \pm 2.03$ & $1.64 \pm 2.38$ & 0.42 \\
Median adiponectin $>1.81$ mmol/l, \% & 50.3 & 56.7 & 0.38
\end{tabular}

Figures in parentheses are percentages.

\section{Discussion}

The most significant contribution of this study was to document that a high proportion of small, dense LDL, particularly if combined with a low adiponectinemia, is significantly associated with early onset of echocardiographic evidence of SVD after BP implantation. To our knowledge, this is the first study to underline that metabolic factors related to the small, dense LDL phenotype are related to SVD. Considering the high prevalence of early echocardiographic signs of SVD (31\%) along with the high number of BPs implanted each year worldwide, as well as the high prevalence of dyslipidemia/dysmetabolic state in the population undergoing aortic valve replacement, this study has potentially important clinical repercussion.

Small, Dense LDL and Structural Valve Degeneration

Although multifactorial, SVD is possibly related to metabolic risk factors and thus potentially modifiable if key targets are identified. Of note, studies have pinpointed that hypercholesterolemia, diabetes and the metabolic syndrome were associated with SVD of BPs $[3,5]$. The present findings suggest that early degenerative failure, identified with the progression of transprosthetic gradient and/or regurgitation, is independently associated
Table 3. Multivariate model for BP dysfunction

\begin{tabular}{lll}
\hline & OR & p value \\
\hline Age $>70$ years old & 1.09 & 0.90 \\
Male gender & 1.12 & 0.74 \\
Body mass index $>30$ & 0.49 & 0.06 \\
$\begin{array}{l}\text { Hypertension } \\
\text { Increased small LDL (\%LDL }<255 \AA)\end{array}$ & 1.70 & 0.14 \\
$\quad$ (higher than median value of $43.2 \%)$ & 1.89 & 0.04 \\
Size of BPs (19-21 mm) & 1.40 & 0.41 \\
\hline
\end{tabular}

with a higher proportion of plasma small, dense LDL. Of particular interest, a recent study has documented a positive association between $\% \mathrm{LDL}_{<255 \AA}$ and the progression rate of the native calcific aortic stenosis [12]. In the same study, $\% \mathrm{LDL}_{<255 \AA}$ was also associated with the content of oxidized LDL in the native valve, which was in turn related to the expression of cytokines and tissue infiltration by inflammatory cells. Albeit calcific aortic stenosis and SVD of BPs are not identical processes, it is possible that common pathways are shared. In this regard, it is possible that small, dense LDLs have a greater ability to infiltrate BPs and to promote inflammation, which may, in turn, 
Table 4. Multivariate model for BP dysfunction according to regurgitation or stenosis

\begin{tabular}{|c|c|c|c|c|}
\hline & \multicolumn{2}{|c|}{ Regurgitation } & \multicolumn{2}{|c|}{ Stenosis } \\
\hline & OR & $\mathrm{p}$ value & OR & $\mathrm{p}$ value \\
\hline Age & 0.98 & 0.47 & 1.00 & 0.82 \\
\hline Gender & 0.88 & 0.77 & 1.59 & 0.27 \\
\hline Body mass index & 0.93 & 0.09 & 0.93 & 0.12 \\
\hline $\begin{array}{c}\text { Small LDL }\left(\% \mathrm{LDL}_{<255 \AA}\right) \\
\text { (higher than median } \\
\text { value of } 43.22 \%)\end{array}$ & 1.01 & 0.24 & 1.00 & 0.49 \\
\hline Adiponectin $^{1}$ & 0.61 & 0.06 & 1.02 & 0.93 \\
\hline
\end{tabular}

Body mass index and the percentage of small LDL were entered in this model as continuous variables.

${ }^{1}$ Adiponectin was entered in the model as a log-transformed variable. In case of SVD due to prosthetic regurgitation, for every 1 -unit increase in adiponectin on log scale, the risk of SVD is reduced by $39 \%$.

trigger degenerative changes. It should be pointed out that small, dense LDLs have a greater rate of oxidation and are highly atherogenic. In fact, clinical studies have documented that small, dense LDLs are associated with the development of coronary artery disease [13-15].

Small, dense LDL is a key feature of abdominal obesity and is often encountered with other metabolic abnormalities such as insulin resistance and hypertriglyceridemia [16]. It is important to mention that a high plasma level of TG is predicting an increased proportion of plasma small, dense LDLs [17]. In fact, among other mechanisms, it is believed that a high TG content in LDL promotes the formation of small, dense LDLs through a process implicating hepatic lipase [18].

\section{Interactions between Adiponectin, Small, Dense LDL and Valve Degeneration}

Adiponectin, a fat-produced peptide, is a multifaceted adipokine with metabolic, anti-inflammatory and antiatherosclerotic properties [19-21]. Experimental studies indicate that adiponectin prevents the formation of foam cells and decreases the production of cytokines upon stimulation with oxidized LDL [22]. In the present study, we found a strong interaction between adiponectin and small, dense LDL. On this score, we documented that patients with a low adiponectinemia and a high proportion of small, dense LDL had a 2.5-fold increase risk of developing early signs of prosthetic dysfunctions. It should be emphasized that a low plasma level of adiponectin is often encountered in viscerally obese patients in whom other metabolic perturbations, such as the presence of the small, dense LDL phenotype, are also present. The question which now arises is whether the interaction between a low adiponectinemia and a high proportion of circulating small, dense LDL is a surrogate marker or an active player in the degenerative process of BPs. Nonetheless, the presence of these two metabolic alterations identifies individuals at higher risk of atherosclerotic complications. In this regard, it is noteworthy that a recent study has documented the presence of foam cells, a hallmark of atherosclerosis, and oxidized LDL in explanted BPs due to SVD [6]. Hence, it is possible that a high proportion/ number of small, dense LDL promotes the accumulation of oxidized LDL in BPs, which, in turn, favors inflammation with ensuing tissue destruction. Of interest, in the present study, we have documented that a low plasma level of adiponectin had a tendency $(p=0.06)$ of being associated with isolated transprosthetic regurgitation. One possible explanation for this finding is that patients with a low adiponectinemia have a higher leaflet inflammatory activity promoting tears and regurgitation. This is in line with our previous studies suggesting that leaflet tears and aortic regurgitation are associated with BP inflammation, activation of metalloproteinase and matrix degradation [6].

\section{Clinical Implications}

The present findings have contributed to underline that a specific group of patients is at high risk of early SVD. It is worth emphasizing that a low adiponectinemia and a high proportion of small, dense LDL are among the key features of visceral obesity. Hence, decreasing the prevalence of visceral obesity would likely translate into lower rates of early SVD. Also, the question which now arises is whether targeting one or both of the identified features in the present study pharmacologically would translate into a lower incidence of SVD. On this score, some pharmacologic agents such as fibrates and peroxisome proliferator-activated receptor- $\gamma$ agonists, which lower the proportion of small, dense LDL and increase adiponectinemia, respectively, might be useful to prevent SVD [23-26]. However, before adopting some recommendation, a randomized trial will be necessary to confirm if it is possible to prevent SVD pharmacologically. Nonetheless, based on the present findings, it is worth recommending the implementation of a lifestyle modification program in patients with a BP in order to possibly reduce the risk of SVD. 


\section{Limitations}

This is a retrospective study. The present association between a high proportion of small, dense LDL and a low adiponectinemia with SVD of BPs does not necessarily imply a cause and effect relationship. However, it has contributed to identify patients at high risk of SVD and cast some light on potential active mechanisms at play in this process. Thus, it opens the perspective that SVD can be prevented. In the present study, SVD has been defined based on echocardiographic findings early after implantation and cannot be used to identify patients who will eventually require surgical replacement of their BPs. Nonetheless, it can be safely proposed that if early echocardiographic signs of SVD are present, these identify a group at risk of further deterioration. In this study, various BPs with different manufacturer labels were included. Owing to the relatively small number of each BP manufacturer label, it is not possible to discriminate whether one type of $\mathrm{BP}$ is at greater or lower risk of failure in relation to the metabolic profile.

\section{Conclusion}

In conclusion, early dysfunction of BP following an aortic valve replacement is frequently encountered in surgical practice. Among the different variables tested in this study, the presence of a high proportion of plasma small, dense LDL and/or a low adiponectinemia was associated with increased risk of developing SVD. Hence, it is possible that active mechanisms participate in the SVD of BPs. While the precise mechanisms involved in SVD remain yet to be elucidated, decreasing the prevalence of dysmetabolic perturbations among patients with an aortic BP would likely translate into a lower rate of early hemodynamic dysfunction of implanted prostheses. Further research is needed to understand and prevent SVD.

\section{Acknowledgements}

This work was supported by a CIHR grant 178768 . N.C. is supported by studentship grants of Fonds de Recherche en Santé du Québec. P.M. is a research scholar from the FRSQ.

\section{References}

1 Schoen FJ, Levy RJ: Founder's Award, 25th Annual Meeting of the Society for Biomaterials, perspectives. Providence, RI, April 28-May 2, 1999. Tissue heart valves: current challenges and future research perspectives. J Biomed Mater Res 1999;47:439465.

-2 Manji RA, Zhu LF, Nijjar NK, Rayner DC, Korbutt GS, Churchill TA, Rajotte RV, Koshal A, Ross DB: Glutaraldehyde-fixed bioprosthetic heart valve conduits calcify and fail from xenograft rejection. Circulation 2006;114:318-327.

3 Nollert G, Miksch J, Kreuzer E, Reichart B: Risk factors for atherosclerosis and the degeneration of pericardial valves after aortic valve replacement. J Thorac Cardiovasc Surg 2003;126:965-968.

-4 Farivar RS, Cohn LH: Hypercholesterolemia is a risk factor for bioprosthetic valve calcification and explantation. J Thorac Cardiovasc Surg 2003;126:969-975.

5 Briand M, Pibarot P, Despres JP, Voisine P, Dumesnil JG, Dagenais F, Mathieu P: Metabolic syndrome is associated with faster degeneration of bioprosthetic valves. Circulation 2006;114:I512-I517

6 Shetty R, Pibarot P, Audet A, Janvier R, Dagenais F, Perron J, Couture C, Voisine P, Despres JP, Mathieu P: Lipid-mediated inflammation and degeneration of bioprosthetic heart valves. Eur J Clin Invest 2009;39:471480 .
7 Antonini-Canterin F, Zuppiroli A, Popescu BA, Granata G, Cervesato E, Piazza R, Pavan D, Nicolosi GL: Effect of statins on the progression of bioprosthetic aortic valve degeneration. Am J Cardiol 2003;92:1479-1482.

-8 Krauss RM, Burke DJ: Identification of multiple subclasses of plasma low density lipoproteins in normal humans. J Lipid Res 1982; 23:97-104.

-9 St-Pierre AC, Ruel IL, Cantin B, Dagenais GR, Bernard PM, Despres JP, Lamarche B: Comparison of various electrophoretic characteristics of LDL particles and their relationship to the risk of ischemic heart disease. Circulation 2001;104:2295-2299.

10 Rainwater DL, Mitchell BD, Comuzzie AG, Haffner SM: Relationship of low-density lipoprotein particle size and measures of adiposity. Int J Obes Relat Metab Disord 1999; 23:180-189.

11 Cheng ML, Kammerer CM, Lowe WF, Dyke B, VandeBerg JL: Method for quantitating cholesterol in subfractions of serum lipoproteins separated by gradient gel electrophoresis. Biochem Genet 1988;26:657-681.

12 Mohty D, Pibarot P, Despres JP, Cote C, Arsenault B, Cartier A, Cosnay P, Couture C, Mathieu P: Association between plasma LDL particle size, valvular accumulation of oxidized LDL, and inflammation in patients with aortic stenosis. Arterioscler Thromb Vasc Biol 2008;28:187-193.
13 Arsenault BJ, Lemieux I, Despres JP, Wareham NJ, Luben R, Kastelein JJ, Khaw KT, Boekholdt SM: Cholesterol levels in small LDL particles predict the risk of coronary heart disease in the EPIC-Norfolk prospective population study. Eur Heart J 2007;28: 2770-2777.

14 Moon JY, Kwon HM, Kwon SW, Yoon SJ, Kim JS, Lee SJ, Park JK, Rhee JH, Yoon YW, Hong BK, Rim SJ, Kim HS: Lipoprotein(a) and LDL particle size are related to the severity of coronary artery disease. Cardiology 2007; 108:282-289.

-15 Kwon SW, Yoon SJ, Kang TS, Kwon HM, Kim JH, Rhee J, Lee SJ, Park JK, Lim JY, Yoon YW, Hong BK: Significance of small dense lowdensity lipoprotein as a risk factor for coronary artery disease and acute coronary syndrome. Yonsei Med J 2006;47:405-414.

-16 Despres JP, Arsenault BJ, Cote M, Cartier A, Lemieux I: Abdominal obesity: the cholesterol of the 21st century? Can J Cardiol 2008; 24(suppl D):7D-12D.

-17 Austin MA, King MC, Vranizan KM, Krauss RM: Atherogenic lipoprotein phenotype. A proposed genetic marker for coronary heart disease risk. Circulation 1990; 82:495-506.

-18 Watson TD, Caslake MJ, Freeman DJ, Griffin BA, Hinnie J, Packard CJ, Shepherd J: Determinants of LDL subfraction distribution and concentrations in young normolipidemic subjects. Arterioscler Thromb 1994;14: 902-910. 
19 Berg AH, Combs TP, Du X, Brownlee M, Scherer PE: The adipocyte-secreted protein Acrp30 enhances hepatic insulin action. Nat Med 2001;7:947-953.

-20 Masaki T, Chiba S, Yasuda T, Tsubone T, Kakuma T, Shimomura I, Funahashi T, Matsuzawa Y, Yoshimatsu H: Peripheral, but not central, administration of adiponectin reduces visceral adiposity and upregulates the expression of uncoupling protein in agouti yellow (Ay/a) obese mice. Diabetes 2003;52: 2266-2273.

21 Okamoto Y, Kihara S, Ouchi N, Nishida M Arita Y, Kumada M, Ohashi K, Sakai N, Shimomura I, Kobayashi H, Terasaka N, Inaba T, Funahashi T, Matsuzawa Y: Adiponectin reduces atherosclerosis in apolipoprotein E-deficient mice. Circulation 2002;106:2767-2770.
22 Tian L, Luo N, Klein RL, Chung BH, Garvey WT, Fu Y: Adiponectin reduces lipid accumulation in macrophage foam cells. Atherosclerosis 2009;202:152-161.

23 Maeda N, Takahashi M, Funahashi T, Kihara S, Nishizawa $\mathrm{H}$, Kishida K, Nagaretani $\mathrm{H}$, Matsuda M, Komuro R, Ouchi N, Kuriyama H, Hotta K, Nakamura T, Shimomura I, Matsuzawa Y: PPARgamma ligands increase expression and plasma concentrations of adiponectin, an adipose-derived protein. Diabetes 2001;50:2094-2099.

24 Combs TP, Wagner JA, Berger J, Doebber T, Wang WJ, Zhang BB, Tanen M, Berg AH, O’Rahilly S, Savage DB, Chatterjee K, Weiss S, Larson PJ, Gottesdiener KM, Gertz BJ, Charron MJ, Scherer PE, Moller DE: Induction of adipocyte complement-related protein of 30 kilodaltons by PPARgamma agonists: a potential mechanism of insulin sensitization. Endocrinology 2002;143:998-1007.
25 Gaw A, Packard CJ, Caslake MJ, Griffin BA, Lindsay GM, Thomson J, Vallance BD, Wosornu D, Shepherd J: Effects of ciprofibrate on LDL metabolism in man. Atherosclerosis 1994;108:137-148.

26 Guerin M, Bruckert E, Dolphin PJ, Turpin G, Chapman MJ: Fenofibrate reduces plasma cholesteryl ester transfer from HDL to VLDL and normalizes the atherogenic, dense LDL profile in combined hyperlipidemia. Arterioscler Thromb Vasc Biol 1996;16:763-772. 